\title{
An update on detergent usage in cryo-EM structure determination of membrane proteins
}

\author{
Edward Pryor ${ }^{1}$, Ritesh Mittal ${ }^{1}$, Ben Travis ${ }^{1}$ \\ ${ }^{1}$ Anatrace Products, LLC, Maumee, OH 43537 (edward_pryor@anatrace.com)
}

The past few years have been revolutionary for the field of single-particle electron cryomicroscopy (cryo-EM), with over $50 \%$ of the total deposited structures being determined since 2014. Currently, there are over 1,600 unique ( $<95 \%$ sequence identity) cryo-EM structures deposited in the PDB, 180 of which are membrane proteins. Although we are witnessing many significant strides in this field, challenges in membrane protein production, purification, and structure determination still persist. These challenges often revolve around the choice of detergent used to solubilize and purify the membrane protein, as it is well established that the detergent used can have drastic effects on the stability and activity of the protein. Thus, choosing the correct detergent is of paramount importance.

We have built a database containing detergent usage data for all unique membrane protein structures deposited in the PDB. Here, we'll present data on which detergents are commonly used in the cryo-EM structure determination of membrane proteins. Specific classes of membrane proteins (e.g. GPCRs) as well as specific structure determination techniques (CryoEM vs X-ray) will be explored, to examine if any particular detergents are better suited for certain protein families or structure determination methods. Additionally, we will highlight examples where the use of fluorinated surfactants, such as Octyl Maltoside and Fos-Choline-8, have been used to improve the vitrification process of membrane proteins. Lastly, we'll look at how new "detergent-free" technologies, such as SMALPs, nanodiscs, and amphipols, are being utilized in this rapidly changing field. 